\title{
La diplomacia chilena hacia los países árabes: entre posicionamiento estratégico y oportunismo comercial
}

\author{
Chilean diplomacy vis-á-vis Arab countries: \\ from strategic positioning \\ to commercial opportunism.
}

\section{Cecilia Baeza* \\ Elodie Brun**}

\section{Resumen}

La diplomacia chilena ha desarrollado en los últimos años una política activa en el terreno comercial hacia los países árabes y, en menor medida, en relación con la cuestión palestina. Nos proponemos en este artículo analizar cuáles son los principales motivos y actores de esta nueva cooperación Sur-Sur, subrayando en particular el papel de la colectividad chileno-árabe.

PALAbRAS ClAvE: política exterior chilena; relaciones chileno-árabes; cooperación Sur-Sur; diáspora árabe-palestina.

* Doctora en Ciencia Política, París. cecilia.baeza.k@gmail.com

** Doctora @ en Ciencia Política, Paris. elodie.brun@yahoo.fr 
Abtract

Over the recent years Chilean diplomacy has developed an active policy in support of increased trade with Arab countries, and to a lesser extent, in relation to the Palestinian issue. In this article, we analyze the key motivations and actors of this new South-South cooperation, stressing the role of the Chilean-Arab community.

KeYwords: Chilean foreign policy, Chilean-Arab relations, South-South cooperation, Arab-Palestinian diaspora.

Recibido el 12 de diciembre de 2011; aceptado el 30 de enero de 2012 
Cecilia Baeza y Elodie Brun • La diplomacia chilena hacia los países árabes...

\section{INTRODUCCIÓN}

La política exterior chilena hacia los países árabes conoce desde unos años un impulso importante. Los tres últimos Presidentes - Ricardo Lagos, Michelle Bachelet y Sebastián Piñera - realizaron todos por lo menos un viaje oficial a un país árabe durante su mandato. Tales viajes presidenciales eran hasta ahora más que escasos en la historia diplomática chilena ya que la última y única visita de este rango al mundo árabe fue la de Salvador Allende a Argelia en 1972. Constituyen un claro indicador de la actividad diplomática chilena que, como lo veremos, se ha centrado en el terreno comercial y en menor medida en la cuestión palestina.

Esta nueva cooperación Sur-Sur se encuentra todavía en su etapa inicial. Si bien estamos lejos de la intensidad de intercambios alcanzada con la región Asia-Pacífico, el acercamiento de Chile hacia los países árabes representa un hecho diplomático suficientemente llamativo como para detener la atención. Existen sin embargo muy pocos estudios. Los que hemos encontrado tratan de la posición de Chile ante la guerra de Irak en 2003 y de la diplomacia chilena frente a la cuestión palestina desde $1947^{1}$ (Fermandois, 2008; Rumié,

\footnotetext{
Esta fecha es recordada por la abstención de Chile en la votación de la resolución 181 de la Asamblea General de las Naciones Unidas del 29 de noviembre 1947, en la que se decidió la partición de Palestina. Chile fue uno de los 10 países que se abstuvieron, mientras que 33 países votaron a favor y 13 lo hicieron en contra.
}

2009). Quedan entonces por explorar muchos aspectos.

Nos proponemos en este artículo analizar cuáles son los principales motivos y actores del proceso iniciado a mediados de la década del 2000. Sostenemos que el acercamiento con los países árabes se inscribe en la tradición diplomática chilena y que la originalidad de estos vínculos proviene más bien de la influencia de ciertos actores sociales. Esto nos permite recordar el papel de los factores internos en la elaboración y conducción de una diplomacia.

Tras una primera parte en forma de balance y puesta en perspectiva histórica de la política exterior de Chile hacia los países árabes ${ }^{2}$, nos interesaremos en el significado estratégico del posicionamiento chileno. Estudiaremos cómo Chile busca ampliar su política exterior en el mundo árabe sin crear tensiones con los Estados Unidos o con Brasil, potencia emergente líder en el acercamiento hacia los países árabes en América del Sur. Examinaremos en particular la posición de Chile respecto de la cuestión palestino-israelí. En la tercera parte se centrará la atención en los intereses económicos y comerciales

2 Definimos los países árabes como los 22 países miembros de la Liga Árabe, organización internacional que reúne a países situados tanto en África como en Asia (incluyendo Palestina). El seguimiento de las relaciones bilaterales con estos países son responsabilidad de la Dirección de Medio Oriente y África en el Ministerio de Relaciones Exteriores. En el Medio Oriente, esta delimitación deja fuera de nuestro estudio a Israel y a Irán. 
en juego en la política exterior chilena en la región, así como en los retos pendientes para profundizar los intercambios. Mostraremos finalmente de manera transversal cómo la evolución de la diplomacia chilena hacia el mundo árabe se vincula estrechamente con el compromiso y la influencia creciente de la diáspora árabe-palestina de Chile respecto de la región de sus antepasados.

\section{UNA NUEVA POLÍTICA EXTERIOR CHILENA HACIA LOS PAÍSES ÁRABES}

Chile desarrolla desde la presidencia de Ricardo Lagos (2000-2005) una diplomacia inusualmente dinámica hacia el mundo árabe. En enero de 2005 el Presidente viajó a Egipto y fue el primer Jefe de Estado chileno en dirigirse a la Liga Árabe. Chile se convirtió así en el segundo Estado no-árabe, junto con Brasil, en integrar la Liga Árabe como miembro observador permanente. Según Luis Palma, entonces encargado de la zona Medio Oriente y África en el Ministerio de Relaciones Exteriores chileno, la visita del Presidente fue minuciosamente preparada, debido a que debía expresar el claro interés del gobierno chileno por estrechar lazos con esta región del mundo (Al Damir, 03/2005: 16). El mismo año, Chile participó en la organización de la primera Cumbre América del Sur-Países Árabes (ASPA) convocada por el gobierno de Lula en Brasilia (MINREL, 25/03/2005). El presidente Lagos asistió en mayo a la cumbre, junto con los mandatarios de 10 otros países sudamericanos y de 22 países del mundo árabe (Vagni, 2005: 101). El Presidente argelino Abdelaziz Bouteflika y el Presidente de la Autoridad Palestina, Mahmud Abbas, aprovecharon esta oportunidad histórica para realizar sucesivamente una visita oficial a Chile en el marco de sus giras regionales. Hasta entonces, nunca un líder palestino de este rango había sido recibido en La Moneda. En menos de un año, Ricardo Lagos había sentado las bases para nuevas relaciones chileno-árabes.

Esta política se vio de hecho confirmada por los sucesores de Ricardo Lagos. El Ministro Secretario General de la Presidencia, José Antonio VieraGallo, visitó los Territorios ocupados palestinos en noviembre de 2008 ( $\mathrm{La}$ Nación, 17/11/2008), mientras que la Presidenta Michelle Bachelet viajó hasta Doha (Qatar) en marzo de 2009 para estar presente en la segunda Cumbre Aspa (Cooperativa, 30/03/2009), y recibió oficialmente el dirigente de la Autoridad palestina en su segunda gira en Sudamérica, en noviembre de 2009 ( $\mathrm{La}$ Nación, 25/11/2009). De manera tal vez menos previsible, la diplomacia del nuevo gobierno de derecha de Sebastián Piñera (2010-2014) se ha inscrito en la continuidad de la política iniciada por la Concertación respecto de los países árabes. En enero de 2011, Chile se sumó a la ola regional de reconocimiento del Estado palestino (MINREL, 07/01/2011). Señal más fuerte aun, apenas dos meses después, el presidente realizó una visita 
presidencial inédita a Palestina en una gira por el Cercano Oriente, donde también pasó por Israel y Jordania $(E l$ Mercurio, 05/03/2011).

Uno de los rasgos más originales de esta política exterior consiste en la implicación de la diáspora árabepalestina de Chile, a la vez como factor de influencia y de legitimación de la diplomacia chilena. Según las instituciones de la colectividad, en Chile los descendientes de inmigrantes palestinos serían más de 350.000. Desde el inicio de la década del 2000, una decena de organizaciones fueron creadas o reactivadas, en reacción al comienzo de la Segunda Intifada en Palestina (Baeza, 2005). Entre ellas destacan la Federación Palestina, entidad creada en 1984 para representar el conjunto de la colectividad ante las autoridades chilenas y la Organización para la Liberación de Palestina (OLP), y la Fundación Palestina Belén 2000, creada en 2000 por los empresarios Mario Nazzal, Alberto Kassis y José Said. Al principio, la Fundación fue creada con una meta precisa y puntual: se trataba de participar en la restauración de la ciudad de Belén (Palestina) para el Jubileo del año 2000, pero con los años fue ampliando sus actividades a donaciones caritativas (principalmente destinadas a Palestina), a la promoción empresarial (tanto entre empresarios de origen árabe en Chile, como entre Chile y el mundo árabe) y a la abogacía (defensa de la causa palestina ante el Gobierno de Chile). Estas organizaciones suelen expresarse sobre las orientaciones de la política chilena en los países árabes, algunas veces criticando las posiciones del gobierno en funciones otras alentando el estrechamiento de las relaciones inter-regionales. Cuentan con enlaces en los partidos políticos (parlamentarios o miembros de gobierno, ellos mismos de ascendencia árabe-palestina ${ }^{3}$ ) que les permiten mantener un diálogo regular y de alto nivel con los actores de la diplomacia chilena.

A nuestro juicio, tal participación de la diáspora árabe-palestina no debe leerse, como la intromisión de un interés particular en la elaboración de la política exterior chilena. Como lo ha demostrado Yossi Shain para el caso estadounidense (1999), las relaciones entre el Estado y las instituciones diaspóricas se caracterizan por su reciprocidad, ya que la influencia de estas últimas depende de la compatibilidad de sus reivindicaciones con la identidad internacional del Estado. En el caso chileno, esto equivale a integrar el «estilo civil-pragmático» que ha dominado en el manejo de la política exterior en período

3 Para el último periodo legislativo por ejemplo, se cuentan los diputados Fuad Chahin Valenzuela (DC), Leopoldo Pérez Lahsen (RN), Jorge Tarud Daccarett (PPD), Joaquín Tuma Zedán (PPD), Mónica Zalaquett Said (UDI), Marcela Sabat Fernández (RN), Jorge Sabag Villalobos (DC), Gustavo Hasbún Selume (UDI), Patricio Hales Dib (PPD) y los senadores Eugenio Tuma Zedán (PPD), Hosaín Sabag Castillo (DC), Carlos Bianchi Chelech (Ind.) y Francisco Chahuan Chahuan (RN). En el ámbito diplomático, puede destacarse a Nelson Haddad, profesor de relaciones internacionales y asesor de la Dirección de Medio Oriente y África del Ministerio de Relaciones Exteriores. 
de democracia, en particular desde los años 1990. Si retomamos la definición de Heraldo Muñoz, este se define principalmente por su énfasis en el derecho internacional y por el reconocimiento práctico de las realidades del poder mundial (Muñoz, 1984). Ambos elementos se encuentran en el discurso elaborado por las principales organizaciones árabespalestinas en Chile: el tema palestino es así sistemáticamente abordado desde el punto de la legalidad internacional y del multilateralismo, mientras que el acercamiento con los demás países árabes es defendido por su dimensión comercial. No existe en Chile, entre las grandes instituciones citadas, posiciones mucho más críticas o radicales frente al orden mundial ${ }^{4}$. Por su lado, el Estado chileno utiliza la presencia árabe-palestina en el país como argumento legitimador para justificar el estrechamiento de las relaciones con el mundo árabe. El discurso de Ricardo Lagos ante los Estados de la Liga Árabe es así característico del uso estratégico de este recurso simbólico:

"Como ustedes lo han recordado, Chile ha recibido a muchos inmigrantes desde hace más de un siglo y alberga una comunidad de origen árabe muy importante que destaca, por su número, como la más grande colonia palestina fuera del Medio Oriente».

Lo cual no significa que discursos de esta índole no estén presentes entre algunos individuos y organizaciones minoritarias de la colectividad. Ver por ejemplo las posiciones anti-imperialistas del Comité Democrático Palestino-Chile, en http:// www.palestinalibre.org/portada_secciones.php?w=Editorial (25/09/2011).
[...] «Junto con los descendientes de otras nacionalidades que llegaron desde Europa y de Asia, los árabes han entregado lo mejor de sí para enriquecer la identidad nacional chilena. Hoy están completamente integrados a nuestra sociedad, aportando con su esfuerzo y trabajo al bienestar de todo el país» (Al Damir, 03/2005: 17).

La presencia en las reuniones realizadas en Egipto del Ministro de Educación Sergio Bitar, el mismo de origen sirio, tuvo también su importancia. El hecho de que el ministro chileno pudiera intervenir «en un perfecto árabe» $(A l$ Damir, 03/2005: 19) creó sin dudas un clima de confianza especial.

Resaltar el carácter novedoso de la política exterior chilena desde el 2005 no significa, sin embargo, que las relaciones de Chile con los países árabes hayan sido hasta entonces nulas, aunque ciertamente más esporádicas que con la región asiática. Los primeros contactos diplomáticos se remontan a 1929 cuando Chile estableció relaciones con Egipto. Luego se iniciaron relaciones con el Líbano y Siria (1945), con Jordania (1954), con Marruecos (1961) y con Argelia (1962). Posteriormente, la Guerra Fría tendió a politizar estas relaciones. Así, el gobierno de Salvador Allende (1970-1973) desarrolló una diplomacia pro-árabe como parte de su acercamiento con el Tercer Mundo y de su retórica antiimperialista. Esta política se desplegó esencialmente en las Naciones Unidas donde Chile votó sistemáticamente a favor de las resoluciones de la Asamblea General en pro 
Cecilia Baeza y Elodie Brun • La diplomacia chilena hacia los países árabes...

de la «causa árabe» (Sharif, 1977: 108). A nivel bilateral, los contactos chilenoárabes fueron en cambio más escasos. En mayo de 1971 el gobierno decidió elevar las relaciones con Libia a nivel de embajada, pero es probablemente con Argelia que la Unidad Popular tejió su relación la más estrecha. En diciembre de 1972, el Presidente Allende fue así recibido «entusiastamente» por el Presidente Boumediene en Argel (Kaufman, 1976: 268). Esta cálida acogida se explica en parte por los vínculos históricos de la izquierda chilena con el Frente de Liberación Nacional (FLN): militantes del pal (Partido AgrarioLaborista) y del FrAP (Frente de Acción Popular) habían creado en 1956 un «Comité pro-autodeterminación de Argelia» que desarrolló hasta 1962 una importante labor de apoyo a la independencia de $\operatorname{Argelia}^{5}$. Fue de hecho un antiguo miembro fundador de este comité, Eduardo Salum Yazigi, quien fue nombrado embajador en Argel en marzo de 1971.

\footnotetext{
En la víspera del $15^{\circ}$ período de sesiones de la Asamblea General de las Naciones Unidas, en agosto de 1960, una importante delegación fue así enviada por el Gobierno Provisional de la República Argelina (GPRA) a Venezuela, Chile y Brasil (1981: 442). La delegación oficial era presidida por Ben Youssef Ben Khedda, quien sería más tarde nombrado presidente del gobierno provisional. La visita a Chile fue un éxito gracias en gran parte a la movilización organizada por el comité. Entre dos y cuatro mil estudiantes asistieron a una conferencia en la Universidad de Chile convocada por el comité. Cf. Entrevista con Eduardo Salum, mayo de 2008.
}

La connotación ideológica de la política exterior del presidente Allende hizo que el gobierno militar establecido por el golpe de Estado del 11 de septiembre de 1973 decidiera en un primer tiempo romper radicalmente con las posiciones pro-árabes de la Unidad Popular. Como lo recuerda Fuad Rumié, el 22 de noviembre de 1974 Chile votó en contra de la resolución 3236 de la Asamblea General de las Naciones Unidas que reconocía el derecho a la autodeterminación del pueblo palestino y de la resolución 3237, que le concedió el estatus de observador a la oLP. El 10 de noviembre de 1975 se abstuvo en la votación de la resolución 3376 de la Asamblea General, que estableció el Comité para el Ejercicio de los Derechos Inalienables del Pueblo Palestino y fue uno de los 32 países en abstenerse en la votación de la resolución 3379 que determinó que el «sionismo [era] una forma de racismo y de discriminación racial» (Rumme, 2009).

Sin embargo, hubo después un período en el cual se intentó mejorar las relaciones de Chile con esta región del mundo (Sharif, 1977: 117-118). En efecto, el gobierno militar estaba buscando apoyos internacionales frente a las reiteradas condenas que sufría en las Naciones Unidas por el tema de los derechos humanos ${ }^{6}$ y a la vez pretendía establecer cooperación económica

6 Según Andrés Sepúlveda, el régimen militar no logró impedir las votaciones. La dictadura intentó la misma estrategia de acercamiento con los países de África negra, pero no obtuvo mucho éxito (1991: 12). 
con países miembros de la OPEP para compensar el alza del barril de crudo provocado por el choque petrolero de 1973. De hecho, la economía chilena dependía mucho de las importaciones de petróleo para su crecimiento. Según la base de datos un Comtrade, en 1975 y en 1976, Arabia Saudita era su primer proveedor de combustibles, representando respectivamente el $31,95 \%$ y el $29,90 \%$ de sus compras. Fue con este propósito que en octubre de 1975 una delegación de alto rango conducida por el Ministro de Hacienda Jorge Cauas y por el Vice-canciller Enrique Valdés Puga viajó al Medio Oriente. Cauas -él mismo de origen palestinopresentaba entonces esa gira como la culminación de «un proceso continuo de mejora de las relaciones amistosas entre [Chile] y el mundo árabe» ( $E l$ Mercurio, 15/08/1975). Se nombraron embajadores en Jordania en 1976 y relaciones diplomáticas se establecieron con Omán en 1976, los Emiratos Árabes Unidos en 1978, Qatar en 1982 y Bahréin en 1983. Este acercamiento con los países árabes se revelaría sin embargo muy precario. La disminución de los precios del petróleo en la década de 1980, el rompimiento en 1978 de los contactos entre el gobierno chileno y la OLP quien decidió apoyar a la oposición clandestina, así como la importancia creciente de Israel como proveedor de armas para Chile a partir de $1977^{7}$,

7 Con la administración de Jimmy Carter (1977-1981) los Estados Unidos empezaron a condenar públicamente las violaciones de los derechos humanos y a presionar debilitaron de manera duradera las relaciones de la Junta militar con los países árabes (Baeza, 2010: 333).

La política exterior chilena hacia el Medio Oriente iniciada por Ricardo Lagos apunta por su parte a un doble objetivo. Participa, en primer lugar, de una tendencia de fondo hacia la «universalización» de la política externa del país (Colacrai y Lorenzini, 2005: 47), la cual cumple a nivel económico un papel de soporte a la diversificación de los mercados para las exportaciones chilenas. En segundo lugar, corresponde a una estrategia de reposicionamiento prudente de Chile en el mapa global, en un contexto de cuestionamiento del orden unipolar y de auge de las potencias emergentes. Es sobre esta segunda dimensión que nos detendremos ahora.

\section{El POSICIONAMIENTO \\ estratégico de Chile a través del Medio Oriente}

\section{Una ecuación que incluye a los Estados Unidos y a Brasil}

La política exterior de Chile hacia los países árabes puede leerse como reveladora del posicionamiento estratégico del

para la restauración de la democracia. Uno de los principales motivos de esta ruptura fue el asesinato en Washington del diplomático chileno Orlando Letelier y uno de sus asociados, un ciudadano de los Estados Unidos, por los servicios de inteligencia chilenos en septiembre de 1976. El Congreso estadounidense votó un embargo sobre las armas contra Chile y el gobierno militar empezó a comprar armas a Israel. 
Cecilia Baeza y Elodie Brun • La diplomacia chilena hacia los países árabes...

país en las relaciones internacionales. El primero de los parámetros es la relación con los Estados Unidos. Chile ha sido y se ha definido como uno de los más fieles aliados latinoamericanos de la primera potencia, pero siempre y cuando esta relación no vaya en contra de los intereses prioritarios del país. Como lo precisó Michelle Bachelet en una conferencia dada en Washington D.C.: «Déjenme empezar por asegurar a los Estados Unidos que pueden contar con Chile como un socio confiable, no un aliado incondicional, pero ciertamente uno abierto y sincero» (Council on Foreign Relations, 25/09/2008). De hecho, la política exterior chilena muestra desde el principio de los años 2000 algunas señales de que el país es capaz de negociar ciertos espacios de autonomía frente a la primera potencia mundial.

Entre aquellas señales se encuentra la posición adoptada por Chile frente a la decisión del gobierno de George W. Bush de intervenir militarmente en Irak para eliminar un supuesto programa de armas de destrucción masiva. La Carta de las Naciones Unidas exige un acuerdo previo del Consejo de Seguridad antes de toda intervención, por lo que un debate estalló en su seno a finales de 2002, mientras Chile era miembro no-permanente de la institución. Empezó una intensa campaña por parte de los defensores de la intervención, Estados Unidos y Gran Bretaña, y de los países que preferían seguir con el diálogo y las inspecciones del Organismo Internacional de la Energía Atómica (OIEA), representados por
Francia en el Consejo. Chile se encontraba en una situación muy delicada. Por un lado, estaba a punto de firmar un Tratado de Libre Comercio (TLC) con los Estados Unidos tras más de diez años de negociación; por el otro, su opinión pública era muy contraria a la intervención y apoyar este proyecto sin autorización del Consejo de Seguridad iba en contra de dos pilares de la diplomacia chilena: el respeto a la legalidad internacional y la preferencia por las soluciones multilaterales ( $E l$ Mercurio, 22/09/2004; 2008). Al final, el presidente Ricardo Lagos propuso una nueva serie de inspecciones para evitar el conflicto armado (El Mercurio, 03/09/2010), pero los Estados Unidos declararon la guerra a Irak antes de la votación de una nueva resolución. Durante el episodio, los representantes estadounidenses se quedaron muy sorprendidos por la decisión chilena de no apoyarlos 8 . Luego de algunos meses de enfriamiento, las relaciones bilaterales volvieron a un buen nivel y se firmó el TLC en junio de 2003 (Muñoz, 2008). Como lo subrayan Manfred Wilhelmy y Roberto Durán, «el proceso de toma de decisiones sobre la guerra de Irak demuestra que el gobierno chileno no ha renunciado a contar con un margen, aunque reducido, de espacio autónomo para la defensa de intereses y principios

8 México, el otro país latinoamericano presente en el Consejo de Seguridad, tampoco apoyó a los Estados Unidos, lo que también fue percibido con una derrota de la primera potencia. 
políticos que considera fundamentales» (Wilhelmy y Durán, 2003: 285).

La participación de Chile en la Cumbre ASPA puede también ser interpretada en este sentido. Si bien la cumbre no tenía como objetivo incomodar a la primera potencia, varios observadores interpretaron la ausencia de representantes estadounidenses como un indicador de la voluntad de autonomía de la región sudamericana respecto del vecino del norte. Según Juan José Vagni, los Estados Unidos pidieron ser observadores del encuentro, pero fue vetada su participación. Siempre según el autor, el Departamento de Estado dio a conocer su preocupación a todas las cancillerías sudamericanas y a las de algunos aliados árabes, presionándolos para que no participaran en la Cumbre9. «A los ojos estadounidenses, la asociación de más de treinta países, algunos de ellos de mucho peso, podría convertirse en un bloque político autónomo» (Vagni, 2009). La declaración de Brasilia, documento aprobado al final de la Cumbre, se alejó de varias de las líneas políticas defendidas por los Estados Unidos en la región, como el párrafo contra las sanciones a Siria

9 Sin embargo, este no era el discurso oficial: de hecho, la Secretaria de Estado estadounidense Condoleezza Rice, tras una reunión a solas con el canciller Celso Amorim en Brasilia, apoyó públicamente la iniciativa: «Nos interesa la interacción entre el mundo árabe y otros países en momentos en que esa región atraviesa profundos cambios. Es una región que necesita reformas económicas, políticas y sociales», señaló Rice (BBC Brasil, 27/04/2005). el que legitima la resistencia palestina contra la ocupación israelí.

Más allá de las fricciones con los Estados Unidos -en realidad, los temores estadounidenses se disiparon completamente con la siguiente Cumbre, mucho más tibia a nivel político-, el proceso ASPa puso de realce el auge de Brasil como potencia regional y global, liderazgo emergente que no es recusado por Chile. En septiembre del 2009, la presidenta Michelle Bachelet describió así a Brasil «como el poder emergente más articulado del sistema internacional y como un actor fundamental en el orden mundial que se está formando» (citado por Sotero, 2010). El posicionamiento de Chile se encuentra por lo tanto entre la aceptación tácita de los Estados Unidos como hegemón y la emergencia de Brasil como actor global y país con el cual el Presidente Piñera tiene la intención de intensificar los vínculos (Yopo Herrera, 2010). Parte de la política chilena en el Medio Oriente debe así leerse como un compromiso entre las limitaciones impuestas por la posición estadounidense y el poder de convocatoria de la diplomacia brasileña.

El mejor ejemplo de este compromiso se encuentra en la forma y el momento en que Chile reconoció al Estado palestino. El 7 de enero de 2011, Chile se sumó a una ola regional en este sentido (MINREL, 07/01/2011). Algunos países latinoamericanos ya habían realizado este reconocimiento oficial - Cuba y Nicaragua, en las semanas que siguieron la proclamación del Estado palestino por la OLP en Argel en noviembre de 1988 y 
Cecilia Baeza y Elodie Brun • La diplomacia chilena hacia los países árabes...

más recientemente Costa Rica en 2008 y la República Dominicana y Venezuela en 2009 (Baeza, 2011). Pero fue la decisión brasileña del 3 de diciembre de 2010 que arrastró un movimiento sin precedentes en América del Sur: Argentina, Bolivia, Ecuador, Chile, Guyana, Perú, Paraguay, Suriname y Uruguay decidieron sucesivamente reconocer el Estado palestino. Esta decisión se tomó a pesar de que Israel se opusiera a tal gesto y de que los Estados Unidos calificaran públicamente el reconocimiento de "contraproducente» (La Nación, 08/12/2010). No obstante, existen matices en las declaraciones que ponen de relieve la política equidistante del gobierno chileno. En efecto, fue el primer país en no mencionar explícitamente las líneas del alto el fuego previas a la Guerra de los Seis Días de junio de 1967 como fronteras del Estado palestino detalle fundamental para los negociadores palestinos quienes buscan revertir la lógica de la colonización israelí en los Territorios ocupados. Esta omisión voluntaria constituye un punto central de la declaración chilena, cuyo objetivo era no irritar a nadie: ni a Brasil y a los palestinos por un lado, ni tampoco a los Estados Unidos e Israel por el otro. Al igual que Chile, Perú y Uruguay adoptaron finalmente la política de no incluir precisiones sobre las fronteras del Estado palestino.

En términos generales, resulta más fácil para Chile alinearse con su socio tradicional cuando se respetan formalmente las reglas internacionales. En marzo de 2011, la cancillería apoyó así la intervención de la OTAN en Libia, la cual se aprobó mediante un voto en el Consejo de Seguridad de las Naciones Unidas. En este caso, no siguió a su vecino brasileño que, como miembro no permanente en el Consejo, decidió abstenerse. Los representantes brasileños se declararon a favor de un bloqueo aéreo contra Libia pero rechazaron cualquier intervención militar, estimando que no sería la mejor solución para resolver el conflicto (Infolatam, 28/02/2011; Nota del MRE brasileño, 28/02/2011).

\section{Una política de equilibrista hacia el conflicto palestino-israelí}

Si se pone en perspectiva la decisión de reconocer el Estado palestino se observa que desde el principio de los años 1990 el gobierno chileno ha intentado mantener buenas relaciones tanto con Israel como con las autoridades palestinas. Su postura oficial se ha limitado a un estricto apoyo a la legalidad internacional: Chile apoya la creación de un Estado palestino soberano en el marco de un acuerdo de paz basado en las resoluciones de las Naciones Unidas y la mayoría de las iniciativas de paz promovidas por la comunidad internacional (Rumié, 2009). En la década de 1990 esto significó primero reequilibrar las relaciones entre las dos partes. Durante el gobierno militar, como ya lo mencionamos, los contactos entre Chile y la OLP se aflojaron, mientras que las relaciones bilaterales con Israel se reforzaron: cuatro de los cinco convenios más importantes entre 
ambos países fueron así firmados entre 1983 y 1988 (BCN Informe, 2011). Los primeros gobiernos de la Concertación se empeñaron por lo tanto en corregir esta asimetría reforzando los vínculos con la oLp. Ello no solamente respondía a una fuerte expectativa por parte de la colectividad árabe-palestina del país sino que se acoplaba al reconocimiento creciente de la olp en el escenario internacional. La Conferencia de Madrid de 1991 y las negociaciones bilaterales iniciadas a partir de 1992 con Israel otorgaron en efecto al movimiento nacional palestino una nueva legitimidad internacional, especialmente ante las principales potencias occidentales.

En marzo de 1990, Jiries al-Atrash fue así el primer representante palestino nombrado por la OLP para abrir una misión oficial en Chile ${ }^{10}$. Pero fue con el nombramiento de Hussein Abdel Khaliq, en mayo de 1992, que las relaciones entre la oLP y el gobierno chileno se fortalecieron a largo plazo. El 17 de diciembre de 1992, una «ceremonia de firma de adhesión» al pueblo palestino se organizó en el Salón de Honor del Congreso Nacional. Era la primera vez que representantes de la colectividad árabe-palestina se reunían con diputados y senadores del Congreso Nacional para expresar su apoyo «a las justas reivindicaciones del pueblo palestino y su anhelo de que se logre una paz justa en el Medio Oriente». Encabezado por el presidente de la Cámara de Diputados, José Antonio

10 http://www.embajadapalestina.cl/embajadores.html (25/09/2011)
Viera Gallo, el evento reunió a cerca de mil inmigrantes palestinos y sus descendientes (La Segunda, 17/12/1992). José Antonio Viera Gallo hizo un llamado al reconocimiento diplomático de la oficina de la olp en Chile. La firma en Washington de los Acuerdos de Oslo ${ }^{11}$ el 13 de septiembre de 1993 aceleró el proceso. El cambio de estatus de la delegación fue obtenido en 1994: la OLP, reconocida como la «Representante del Pueblo Palestino", contó a partir de esa fecha con una oficina de representación con una serie de privilegios e inmunidades diplomáticos (DS 57, RREE, 1994). En 1996, el gobierno chileno suscribió con la olP un «Memorándum de Entendimiento relativo a la Cooperación Científica, Técnica, Cultura y Educativa Chileno-Palestina» (DTO 1866, RREE, 1996). En abril de 1998, Chile fue el primer país del continente en abrir una delegación ante la Autoridad Palestina en Ramallah (La Tercera, 17/06/1998). Paralelamente, a partir de 1990 Chile

11 Los Acuerdos de Oslo fueron una serie de pactos negociados entre el gobierno israelí y la OLP. Fueron firmados en 1993 como primera etapa del proceso de paz entre palestinos e israelíes. El proceso preveía la retirada progresiva de las fuerzas israelíes de la Franja de Gaza y Cisjordania, así como el derecho de los palestinos al autogobierno en esas zonas a través de la creación de una Autoridad palestina. El gobierno palestino duraría cinco años de manera interina, durante los cuales el estatus sería renegociado (a partir de mayo de 1996). Las cuestiones acerca de Jerusalén, los refugiados, los asentamientos israelíes, la seguridad y las fronteras exactas quedarían para la última fase de la negociación. 
Cecilia Baeza y Elodie Brun • La diplomacia chilena hacia los países árabes...

votó sistemáticamente en favor de las resoluciones de la Asamblea General de las Naciones Unidas en defensa de los derechos del pueblo palestino ${ }^{12}$.

El clima de optimismo internacional acerca de un posible acuerdo de paz definitivo entre Israel y los palestinos empezó sin embargo a declinar a finales de los años 1990 con el deterioro de la situación a nivel local, que en septiembre de 2000 desembocó en la segunda Intifada. El avance de la colonización israelí en los Territorios palestinos, la imposibilidad de llegar a un acuerdo sobre los refugiados palestinos, así como el recrudecimiento de la violencia, tanto por parte del ejército israelí como de la resistencia palestina, polarizaron nuevamente a la comunidad internacional respecto de las responsabilidades en el fracaso del proceso de paz. Si bien el surgimiento de planes de paz había generado amplio consenso internacional, el regreso de la inestabilidad en la región volvió a dividir los actores. Los Estados Unidos, en particular, tradicionales aliados de Israel en la región, apoyaron la posición del Estado hebreo atribuyendo la responsabilidad

12 Chile votó, entre otras, a favor de las resoluciones de la Asamblea General 44/2, de 1989 y 46/76 de 1991, que condenaron las prácticas de Israel contra los palestinos participantes en la Intifada; 44/174 de 1989 , y 46/162, de 1991, que rechaza las acciones israelíes para cambiar la composición demográfica de los territorios ocupados; 47/82 de 1992, que reafirmó el derecho del pueblo palestino a la independencia, integridad nacional y territorial y 54/37, de 1999 , que declaró la nulidad de la jurisdicción israelí sobre Jerusalén (RUMIÉ, 2009). del bloqueo de las negociaciones a la parte palestina.

La década del 2000 fue por lo tanto más delicada para los diplomáticos chilenos. Hasta entonces, los representantes políticos podían en efecto a la vez perseguir sus objetivos económicos en el Medio Oriente y profundizar sus relaciones con la OLP sin perjudicar sus relaciones con sus socios tradicionales de Occidente. Desde la segunda Intifada, en cambio, cada gesto hacia la parte palestina ha requerido une contraparte. La línea equidistante buscada por los gobiernos chilenos implicó dar más pruebas a la parte israelí para complacer a su aliado estadounidense. De manera significativa, cada visita oficial chilena a los Territorios palestinos en los años 2000 incluyó sistemáticamente a Israel, lo que no es el caso de otros países, como Brasil por ejemplo. La visita inédita en marzo de 2005 del Ministro de Relaciones Exteriores Ignacio Walker a los Territorios palestinos fue así equilibrada por su visita, también histórica, a Israel. El modelo se repitió en noviembre de 2008 con la doble visita del Ministro Secretario General de la Presidencia, José Antonio Viera-Gallo, y en marzo de 2011 con el presidente Piñera. Durante su estadía en el Medio Oriente, Sebastián Piñera declaró: "Chile ha reconocido a Palestina porque al igual que siempre hemos pensado que Israel tiene el derecho a vivir en un país con fronteras permanentes, reconocidas por la comunidad internacional con el objeto de poder desarrollarse, los palestinos 
también tienen derecho a un país libre y democrático» (Efe, 06/03/2011). Por lo demás, la relación entre Chile y la Autoridad palestina se reorientó sobre aspectos humanitarios - con la firma por ejemplo de un «Programa de Cooperación Médica Chilena-Palestina» en el $2008^{13}$-, mientras que con Israel, el gobierno inició conversaciones para la suscripción de un TLC ${ }^{14}$.

Este posicionamiento equidistante se asemeja a un ejercicio de equilibrista. Las demandas de las organizaciones comunitarias de descendientes palestinos sobre este tema han sido en efecto particularmente fuertes desde el inicio de los años 2000. Como se dijo, las organizaciones árabe-palestinas se encuentran movilizadas desde hace unos diez años y participan activamente en el debate sobre el manejo de la política exterior. Exigen que el gobierno chileno se muestre más resuelto en pedir que Israel cumpla con el derecho internacional. Cuentan en este sentido con amplios apoyos en el Congreso Nacional, tanto en la Cámara de Diputados como en el Senado. Como lo subraya Fuad Rumié (2009), el Congreso ha demostrado un interés inigualado por el tema: en el periodo legislativo 20062010 , se aprobaron seis proyectos de acuerdo referentes al conflicto palestinoisraelí, cuatro de los cuales tomando una clara posición en favor de los derechos

13 http://chileabroad.gov.cl/palestina/relacion-bilateral/acuerdos-y-tratados-bilaterales/ (25/09/2011).

14 http://chileabroad.gov.cl/israel/relacionbilateral/comercio-relaciones-bilaterales/ (25/09/2011). palestinos (RUMIE, 2009). Las voces parlamentarias se hacen cada vez más escuchar, como por ejemplo para pedir el cese de la invasión israelí a la Franja de Gaza iniciada en diciembre de 2008 (El Mercurio, 07/01/2009) o para condenar el ataque a la Flotilla Humanitaria que llevaba ayuda a Gaza en el 2010 (El Mercurio, 01/06/2010). Destacar este apoyo no significa que los sectores pro-israelíes no estén movilizados. La comunidad judía de Chile así como la embajada de Israel también tratan de difundir sus argumentos. Por ejemplo organizan viajes a Israel en los cuales se mezclan visitas a lugares turísticos y actos oficiales, destinados a demostrar la validez de la política del Estado hebreo. Desde el 2006, más de 80 parlamentarios chilenos han sido convidados a participar en estas giras (Rumié, 2009). Estas actividades de lobbying hacia el Congreso son particularmente reveladoras. Si bien la institución legislativa no es el ente responsable de la conducción de la política exterior chilena, sus declaraciones públicas participan en la definición del posicionamiento nacional en las relaciones internacionales e influyen en la toma de decisiones. La secuencia que llevó al reconocimiento del Estado palestino por parte de Chile en enero de 2011 -desde las declaraciones de la Federación Palestina de Chile hasta el activismo de los parlamentarios (Cooperativa, 20/12/2010)-ilustra bien, a nuestro juicio, los mecanismos de influencia de la sociedad civil sobre la política exterior chilena. 
Cecilia Baeza y Elodie Brun • La diplomacia chilena hacia los países árabes...

La posición de Chile respecto del conflicto palestino-israelí resulta finalmente de un conjunto de factores, tanto externos como internos. A nivel interno, el Estado teme por encima de todo «importar el conflicto en Chile», a través de las colectividades árabe y judía politizadas del país. Si bien siempre ha prevalecido una buena convivencia entre estas colectividades socialmente muy cercanas, pequeños roces entre ambas alimentan al respecto desde unos años ciertas inquietudes -a veces exageradas. Respecto de los factores externos, Chile trata de complacer tanto a su aliado estadounidense como a sus nuevos socios árabes. Este último factor es probablemente decisivo: en efecto, los mandatarios chilenos saben que su influencia en la cuestión palestina es reducida, pero su empeño en dar a conocer su postura nos lleva a pensar que existen otros motivos para justificar su acercamiento a la posición árabe, en particular de orden económico.

\section{El Medio Oriente como NUEVA FRONTERA ECONÓMICA- COMERCIAL PARA CHILE}

El acercamiento chileno al Medio Oriente: afinidades estructurales

Chile, por sus características estructurales (grandes recursos naturales y una economía de pequeño tamaño), ha consagrado históricamente gran parte de su política exterior a la dimensión comercial de sus relaciones externas. Diversificar sus mercados de exportación ha siempre constituido un objetivo y una variable de primer orden a la hora de establecer relaciones diplomáticas, incluso con los países en desarrollo. Esta preponderancia económica se traduce en la estructura del Ministerio de Relaciones Exteriores: en el 2008, la Dirección General de Relaciones Económicas Internacionales (DIRECON) -entidad encargada de los objetivos económicos- contaba así para los Estados del Sur con un número equivalente de diplomáticos al de la Dirección General de Política.

El acercamiento de Chile con los países árabes forma parte de esta estrategia de ampliación del comercio exterior chileno. Chile se ha construido un perfil externo bastante diversificado en términos de socios (Cuadro ${ }^{\circ} 1$ ). La década del 1990 fue marcada por la voluntad de ascender el nivel de las relaciones con los países del Asia-Pacífico (Wilhelmy y Durán, 2003: 282-283). Los resultados fueron vertiginosos: en 2009, el continente asiático absorbió casi el $44 \%$ de las exportaciones chilenas y el $23 \%$ de sus importaciones (Cuadro ${ }^{\circ} 1$ ). Con el Medio Oriente en cambio, las relaciones económicas se sitúan aún en un plano de potencialidad.

Calculando a partir de las cifras del cuadro ${ }^{\circ} 2$ los intercambios con los países árabes del Medio Oriente crecieron significativamente desde la transición democrática: las exportaciones aumentaron un $79 \%$ entre 1990 y 2009 y las importaciones subieron un $136 \%$. Los resultados hubieran sido aún más im- 
portantes si no fuera por la crisis económica internacional que estalló en 2008 y que afectó de manera significativa a las relaciones comerciales de Chile con estos países entre 2008 y 2009. Los in- tercambios todavía no son muy estables, como lo muestran las variaciones de las exportaciones y de las importaciones así como la inestabilidad de los productos intercambiados (Cuadro $\left.{ }^{\circ} 4\right)$.

Cuadro $N^{\circ} 1$ : Relaciones comerciales de Chile con el mundo en 2009

\begin{tabular}{c|ccccc|c} 
& $\begin{array}{c}\text { Estados } \\
\text { Unidos }\end{array}$ & UE & $\begin{array}{c}\text { América Latina } \\
\text { y el Caribe }\end{array}$ & Asia & $\begin{array}{c}\text { Resto del } \\
\text { mundo }\end{array}$ \\
\hline Exportaciones & 11,3 & 16,9 & 16,3 & 43,7 & 11,8 \\
\hline Importaciones & 16,8 & 14,8 & 29,7 & 22,5 & 16,2
\end{tabular}

Fuente: CEPAL (SIGCI)

Cuadro N²: Auge de las relaciones comerciales de Chile con el Medio Oriente ${ }^{15}$

\begin{tabular}{c|cc|cc|cc|cc|c|c|} 
& \multicolumn{2}{|c|}{1990} & \multicolumn{2}{c|}{2000} & \multicolumn{2}{c|}{2005} & \multicolumn{2}{c|}{2008} & \multicolumn{2}{c|}{2009} \\
\cline { 2 - 11 } & Exp. & Imp. & Exp. & Imp. & Exp. & Imp. & Exp. & Imp. & Exp. & Imp. \\
\hline \multirow{2}{*}{$\begin{array}{c}\text { Medio Oriente } \\
\text { (en US\$) }\end{array}$} & 123 & 17 & 234 & 49 & 212 & 14 & 320 & 144 & 221 & 42 \\
& 363 & 825 & 074 & 703 & 693 & 136 & 778 & 600 & 173 & 101 \\
\hline $\begin{array}{c}\text { Medio Oriente / } \\
\text { Mundo } \\
\text { (en \%) }\end{array}$ & 1,49 & 0,25 & 1,31 & 0,30 & 0,52 & 0,04 & 0,49 & 0,23 & 0,42 & 0,10 \\
\hline
\end{tabular}

Fuente: UN Comtrade

Si en términos nominales, los intercambios aumentan entre 1990 y 2009 , no sucede así en términos relativos. Por ahora la participación del Medio Orien-

15 No se incluyen Irán, ni Israel, ni Turquía (clasificada con los países europeos por la diplomacia chilena). En la base de datos uN Comtrade aparecen las cifras para los Territorios palestinos, cuando los países los entregan; en el caso chileno no aparecen (puede ser porque se mezclan con las de Israel). te en el comercio internacional de Chile se mantiene en un nivel extremadamente bajo. Sin embargo, Chile se beneficia de una balanza comercial muy favorable. La diplomacia chilena, cuando se interesa en esta región, intenta por lo tanto crear nuevos canales de comercio más que gestionar flujos ya existentes.

La lista de los principales socios comerciales árabes de Chile (Cuadro $n^{\circ} 3$ ) corresponde estrechamente a los 
blancos de la diplomacia chilena. Así, las visitas presidenciales honraron socios comerciales importantes: Arabia Saudita, los Emiratos Árabes Unidos, Egipto y Qatar. El Ministerio se ha enfocado en el Península Arábiga, también porque es en esa región donde se encuentran sus principales compradores árabes. Por ejemplo en 2009, se abrió una embajada en Dubai, precedida el año anterior por una oficina de ProChile, la agencia estatal de fomento a las exportaciones (Boletines del Minrel, 18/10/2009; Boletines de la DIRECON, 05/06/2008). Desde entonces, varias empresas chilenas participan en la Gulfood, importante feria comercial de la región y ProChile incluyó a Dubái en su programa «Semana de Chile», serie de actividades desarrolladas para dar a conocer los productos chilenos (Boletines de la DIRECON, 01/03/2007; 21/10/2009). La DIRECON propuso entablar las negociaciones para concretar un TLC con el Consejo de Cooperación del Golfo. Este Consejo está compuesto por Arabia Saudita, Bahréin, Qatar, los Emiratos Árabes Unidos, Kuwait y Omán. Las discusiones, comenzadas en el 2008, siguen su camino.

Cuadro $n^{\circ}$ 3: Principales socios de Chile en el Medio Oriente

\begin{tabular}{|c|c|c|c|c|}
\hline & \multicolumn{2}{|c|}{2008} & \multicolumn{2}{|c|}{2009} \\
\hline & Exportaciones & Importaciones & Exportaciones & Importaciones \\
\hline 1 & $\begin{array}{l}\text { Emiratos } \\
\text { Árabes Unidos }\end{array}$ & Arabia Saudita & Arabia Saudita & Qatar \\
\hline 2 & Arabia Saudita & Qatar & $\begin{array}{c}\text { Emiratos } \\
\text { Árabes Unidos }\end{array}$ & Arabia Saudita \\
\hline 3 & Egipto & Egipto & Egipto & Egipto \\
\hline 4 & Siria & $\begin{array}{c}\text { Emiratos } \\
\text { Árabes Unidos }\end{array}$ & Argelia & $\begin{array}{c}\text { Emiratos } \\
\text { Árabes Unidos }\end{array}$ \\
\hline 5 & Omán & Marruecos & Kuwait & Marruecos \\
\hline
\end{tabular}

\section{Fuente: UN Comtrade}

El alto nivel de los precios del petróleo en los últimos años ha realzado el potencial de estos países como socios comerciales. Grandes consumidores de cobre y sobre todo de productos agrícolas, los países del Medio Oriente son importado- res netos de los commodities producidos por Chile. Solo recordemos que la diversificación de la economía chilena para salir de la dependencia del cobre pasa por el desarrollo de la cadena agro-industrial (uva, madera, manzanas, etc.). 
Estudios Internacionales I7I (2012) • Universidad de Chile

Cuadro $n^{\circ} 4$ : Principales productos intercambiados entre Chile y sus socios árabes

\begin{tabular}{|c|c|c|}
\hline Productos & \multicolumn{2}{|c|}{ Proporción (en \%) } \\
\hline Importaciones chilenas desde: & 2008 & 2009 \\
\hline \multicolumn{3}{|c|}{ Arabia Saudita } \\
\hline Gas natural & 83,44 & NF \\
\hline Materiales plásticos & 10,40 & 86,10 \\
\hline \multicolumn{3}{|l|}{ Qatar } \\
\hline Gas natural & 99,99 & 99,80 \\
\hline \multicolumn{3}{|c|}{ Emiratos Árabes Unidos } \\
\hline Equipamiento eléctrico & 31,77 & 0,86 \\
\hline Cristalería & 14,14 & 6,19 \\
\hline Materiales plásticos & 16,68 & 21,90 \\
\hline Hilo de textil & 6,05 & 19,85 \\
\hline Exportaciones chilenas a: & 2008 & 2009 \\
\hline \multicolumn{3}{|c|}{ Arabia Saudita } \\
\hline Madera (para construcción) & 36,22 & 21,59 \\
\hline Cobre refinado & NF & 28,64 \\
\hline \multicolumn{3}{|l|}{ Qatar } \\
\hline Madera (para construcción) & 67,24 & 66,57 \\
\hline \multicolumn{3}{|c|}{ Emiratos Árabes Unidos } \\
\hline Madera (para construcción) & 48,49 & 21,18 \\
\hline Frutas frescas & 23,49 & 46,59 \\
\hline
\end{tabular}

Fuente: UN Comtrade (a 1 dígito de la Clasificación uniforme del comercio internacional de Naciones Unidas, REV.1) 
Cecilia Baeza y Elodie Brun • La diplomacia chilena hacia los países árabes...

Por lo demás, gracias a los ingresos del petróleo, varios países árabes se están convirtiendo en importantes inversores internacionales y Chile puede tener interés en atraerlos. El crecimiento económico sostenido, el desarrollo de las clases medias, la estabilidad política del país y la seguridad de las inversiones extranjeras son fuertes argumentos para atraer capitales ${ }^{16}$. Así, en octubre de 2009 se firmó un acuerdo de cooperación económica con los Emiratos Árabes Unidos (Boletines de la DIRECON, 20/10/2009). En julio de 2010, el primer ministro kuwaití, Sheikh Nasser Al-Mohammad Al-Ahmad Al-Sabah, realizó una gira oficial por Latinoamérica. En Chile, el alcalde de Santiago le entregó las llaves de la ciudad como símbolo de su papel en el estrechamiento de las relaciones kuwaití-chilenas ${ }^{17}$.

No obstante, las relaciones financieras entre Chile y Medio Oriente todavía se encuentran en un nivel incipiente. Una de las razones es la falta de canales institucionales financieros: en los países del Golfo Arábigo no hay ningún banco chileno presente y a su vez los bancos árabes no están representados en Chile. Según la DiRecon, entre 1990 y 2009, «las incursiones de capitales chilenos en los países del Oriente Medio, de acuerdo con la información disponible, han sido relativamente acotadas y si bien

16 Ver en particular: «Building A Bridge to the Middle East», http://www.alternativelatininvestor.com/47/emerging-markets/ building-a-bridge-to-the-middle-east.html (25/09/2011).

17 ht t p://www.pm.gov.kw/cv.asp (25/09/2011). se han registrado proyectos en países como Irán y Yemen, en la actualidad se tiene conocimiento de presencia de inversiones chilenas solo en Egipto y en los Emiratos Árabes ${ }^{18}$ » (Boletines de la DIRECON, 10/2010). Los proyectos en estos dos últimos países suman 127 millones de dólares.

Un detalle llama la atención: las inversiones chilenas en Egipto se dedican a proyectos de prospección y explotación de yacimientos petrolíferos a través de concesiones a la empresa ENAP-SIPETROL. El petróleo producido se destina a Europa porque el costo de transporte hacia Chile es muy alto. Esta situación recuerda las dificultades con que tropiezan los países en desarrollo a la hora de aumentar sus intercambios, debido a la debilidad de las rutas comerciales. Las recientes aperturas de canales de comunicación marítimas y aéreas entre Brasil y el Medio Oriente (Dubai-São Paulo; Doha-São PauloBuenos Aires) son señales en este sentido, así como los proyectos de corredores bioceánicos que abrirían la fachada atlántica a Chile. Por todas estas variables, esta región se dibuja todavía como una nueva frontera para las exportaciones chilenas.

\section{El despertar de los empresarios chilenos-palestinos}

La diplomacia chilena hacia el Medio Oriente presenta un perfil muy dinámico también debido a que encuentra

18 Nueve países concentran un 90,7\% de la inversión chilena en el exterior: Argentina, Brasil, Perú, Colombia, Estados Unidos, México, Australia, Uruguay y Venezuela. 
eco en el sector empresarial. Existe en efecto cierto interés entre los empresarios y gremios chilenos por esta región (incluyendo Turquía), aunque menor que por Asia ${ }^{19}$. Los propios actores reconocen que esta atención es nueva. Por ejemplo, un antiguo director de la DIRECON explica que la Dirección intentó organizar una misión comercial al Medio Oriente a principios de los años 2000 pero que no se concretó por la falta de interés que existía entonces en el sector privado ${ }^{20}$. En cambio, un proyecto similar se logró en 2007 cuando ProChile y el Comité de Inversiones Extranjeras (Cinver) presidieron una misión compuesta por once empresas nacionales a Emiratos Árabes Unidos, Arabia Saudita y Qatar (Boletines de la DIRECON, 26/01/2007). El año siguiente, la entonces directora de ProChile, Alicia Frohmann, viajó a Dubai, Israel y a los territorios palestinos (Boletines de la DiRECon, 05/06/2008).

El perfil de los empresarios activos en este acercamiento chileno al Medio Oriente no es indiferente para entender cómo surge su interés por la región. En efecto, la gran mayoría son de origen árabe, generalmente palestinos. Muchos pertenecen a familias de larga

19 Cf. Entrevistas con representantes de la Asociación de Bancos e instituciones financieras (ABIF), de CODELCO, de la Sofofa y de la Confederación de la Producción y del Comercio (CPC) llevadas a cabo por Élodie Brun entre junio y octubre de 2008 en Santiago de Chile.

20 Entrevista con un antiguo director de la DIRECON, realizada por Élodie Brun el 25 de octubre de 2010. trayectoria empresarial, a menudo ligadas al rubro textil hasta que la apertura económica del país en la segunda mitad de los años 1970 provocó un declive brutal del sector y su reconversión económica (en los ámbitos financiero, inmobiliario y de los medios de comunicación principalmente). La intuición de que su identidad diaspórica podría desempeñar un papel positivo para crear puentes económicos entre las dos regiones empezó a cobrar fuerza en la segunda mitad de los años 2000. La temática de los países emergentes y de las nuevas relaciones Sur-Sur alentó los empresarios en este sentido.

En noviembre de 2007, un «grupo de visionarios empresarios chilenos de origen árabe» creó así el «Consejo Empresarial Chileno-Árabe» (ChileArab) bajo el alero de la Sociedad de Fomento Fabril (SOFOFA), «con el objetivo de acercar empresarialmente a Chile y los Países Árabes en términos de intercambio comercial y oportunidades de inversión». El directorio de la organización cuenta con importantes figuras del empresariado chileno. Su presidente es José Said, presidente del Banco Bilbao Vizcaya (BBva), del Parque Arauco y director de Embotelladora Andina - una de las principales fortunas del país. La descripción de ChileArab sigue: «Además de pertenecer a una de las instituciones más emblemáticas del sector privado chileno, su estrecha relación con Cancillería, y sus vínculos con la red árabe Young Arab Leaders $^{21}$, garantizan a ChileArab la más

21 Se trata de una organización nacida bajo el auspicio de las monarquías del Golfo para promover «la educación y el espíritu 
Cecilia Baeza y Elodie Brun • La diplomacia chilena hacia los países árabes...

amplia red de contactos empresariales en Chile y el mundo árabe tanto en el sector privado como en el público, fortaleciendo su misión de constituirse en el puente natural entre ambos mercados. ChileArab aporta valor al país en términos de apertura y desarrollo de mercados complejos pero con un importante potencial de consumo y flujo de capitales, a la vez que presenta a Chile como plataforma de inversiones para la región ${ }^{22} »$. Los empresarios no hacen misterio de sus vínculos con la DIRECON.

El contexto en que nace ChileArab habla de sus relaciones privilegiadas con el mundo político. La organización surgió a raíz de un gran seminario empresarial realizado el 30 de octubre 2007 en el Hotel Hyatt, a iniciativa de la Fundación Palestina - Belén 2000, y bajo los auspicios de los Young Arab Leaders (Al Damir, 10/2007). El poder de convocatoria de los empresarios de origen palestino es de alto nivel: estaban presentes la Presidenta Michelle Bachelet, el Ministro de Relaciones Exteriores Alejandro Foxley, el Vicepresidente de la Cámara de Comercio Árabe-Brasileña (ССAB) Paulo Atallah, y Carlos Slim, empresario mexicano de origen libanés, cuya familia era en aquel momento la segunda fortuna mundial según Forbes ${ }^{23}$. Verdadero «Rey Midas» (La Nación, 4/11/2007), Carlos Slim

empresarial». http://www.yaleaders.org/ en/ (25/09/2011).

22 «Quiénes somos», http://www.chilearab. cl/nosotros.html (25/09/2011).

$23 \mathrm{http} / /$ www.lanacion.com.ar/899647-slimla - s egunda-fortuna-de l-mundo (20/01/2012). era el invitado estrella del seminario, en el que participaron más de 900 personas. Su presencia estaba allí para significar la fuerza y la influencia de las redes árabes en América Latina. La presencia de Michelle Bachelet en este seminario dice también mucho acerca de las nuevas redes de influencia de estos hombre de negocios, dispuestos a cortejar a la izquierda. La reconciliación del Partido Socialista de Chile con el negocio de ascendencia árabe se inició a fines de 1990: en la campaña presidencial de 1999, el candidato Ricardo Lagos había sostenido una reunión en el Club Palestino donde acompañado del entonces embajador de Palestina Hussein Abdel Khaliq fue a pedir públicamente "perdón» a la comunidad por la ocupación y la expropiación de fábricas textiles sufridas bajo la Unidad Popular (La Segunda, 19/4/1999), señal simbólica de que los tiempos habían definitivamente cambiado.

Desde hace algunos años, ChileArab está en el primer plano del estrechamiento de las relaciones comerciales entre Chile y los países del Golfo Arábigo. Su director ejecutivo, Jorge Daccarett, representó al sector privado chileno durante el encuentro empresarial de la segunda cumbre ASPA de Doha en 2009 (ANBA, 25/03/2009). En la Cumbre de las Inversiones Árabes-Latinoamericanas en abril de 2010 en Doha y en Abu Dabi, el consejo empresarial volvió a organizar una importante delegación chilena, en la que destacó Salvador Said, presidente ejecutivo del grupo Said S.A. El activismo de los empresarios chilenopalestinos va más allá del marco nacional. 
También los encontramos en la fundación en el 2009 del Gulf Latin America Leaders Council (GLCC), organización empresarial bi-regional cuyo objetivo es promover «alianzas estratégicas e inversiones entre el Consejo de Cooperación para los Estados Árabes del Golfo Pérsico y América Latina. [...] GLCC busca construir relaciones económicas y personales entre los líderes de más alto nivel y responsables de las decisiones tanto del sector privado y el sector público ${ }^{24}$. En realidad, la principal meta de la organización es guiar las inversiones de los fondos soberanos del Golfo en América Latina. De manera significativa, encontramos a Jorge Daccarett Bahna, Director ejecutivo de ChileArab, también como Secretario del GLCC.

\section{Conclusión}

Como hemos planteado desde la introducción, la especificidad más destacable del acercamiento de Chile a los países árabes radica en la participación de la colectividad chileno-árabe. Esta ha sido fundamental en el proceso para generar relaciones de confianza y de afinidad cultural. La dimensión diaspórica le añade en efecto al nivel intergubernamental una profundidad sociológica que puede aseguradamente darle durabilidad a la política exterior chilena.

Las nuevas actividades chilenas hacia los países árabes que hemos analizado abren oportunidades inéditas de cooperación Sur-Sur para este país suramericano.

24 http://www.gllc.org/english/?page_id=2 (25/09/2011).
Esta diplomacia se ha centrado en el refuerzo de los vínculos comerciales interregionales y en el apoyo a la aplicación del derecho internacional en la región. En este sentido, respeta sus fundamentos tradicionales, entre los cuales el de mantener buenas relaciones con todos sus socios a pesar de las tensiones entre ellos. Los representantes chilenos insisten en demostrar que el acercamiento de Chile a los países árabes no apunta a tener un carácter contra-hegemónico, sino más bien a profundizar la tendencia a la universalización de sus relaciones internacionales iniciada hace ya unas décadas.

Resulta no obstante difícil penetrar el mundo árabe y el Medio Oriente sin comprometerse de una forma u otra con las cuestiones políticas que los afectan, como lo demostró el caso brasileño. Frente a estas dificultades, Chile ha tratado de mantener su posición de mero defensor de la legalidad internacional, respaldándose alternativamente en el planteamiento de su aliado tradicional los Estados Unidos - o el de la potencia emergente sudamericana que es Brasil. En un contexto de profundos cambios en el mundo árabe, se sabrá más pronto que tarde a qué beneficios o cuáles tensiones pueden conducir esta política de equilibrios, y si es posible que se mantenga.

\section{Bibliografía}

Baeza, Cecilia (2005) «Les Palestiniens du Chili: De la conscience diasporique à la mobilisation transnationale», Revue d'études palestiniennes, $\mathrm{N}^{\circ} 95$, Printemps, pp. 51-87

Baeza, Cecilia (2010) Les Palestiniens d'Amérique Latine et la cause palestinien- 
Cecilia Baeza y Elodie Brun • La diplomacia chilena hacia los países árabes...

ne (Chili, Brésil, Honduras -1920-2010), Tesis de Doctorado en Ciencia política (Relaciones internacionales), París, Instituto de Estudios Políticos 589 pp.

BAEZA, Cecilia (2011), «O reconhecimento do Estado palestino: origens e perspectivas», Boletim Meridiano 47, Julho-Agosto.

Barrata, Robert Thomas (1989) «The PlO in Latin America», en Norton, Augustus R., Greenberg, Martin H. (comp.), The International Relations of the Palestine Liberation Organization, Carbondale/ Edwardsville, Southern Illinois University Press, , pp.166-195.

Снікн, Slimane (1981), L'Algérie en armes, ou le temps des certitudes, Paris, Economica, $511 \mathrm{pp}$.

Colacrai Miryam y María Elena Lorenzini (2005), «La política exterior de Chile: ¿excepcionalidad o continuidad? Una lectura combinada de «fuerzas profundas» y tendencias", confines, (1/2) agostodiciembre, p.p 45-63

Fermandois, Joaquín (2005), Mundo y fin de mundo. Chile en la política mundial 19002004, Santiago, Universidad Católica de Chile, 638 pp.

Fermandois, Joaquín (2008), "Chile y la Guerra de Irak», Bicentenario, vol.7, n²1, pp.45-78.

Fuentes, Julio, Claudia F. y Francisco, Aravena (2010), "Chile and the United States 20002009: From Elusive Friendship to Cooperative Friendship», en Domínguez, Jorge I., Fernández de Castro, Rafael (comp.), Contemporary US-Latin American Relations: Cooperation or Conflict in the $21^{\text {st }}$ Century?, New York, Routledge, pp.142-163.

Glick, Edward (1959), "Latin America and the Palestine Partition Resolution", Journal of Inter-American Studies, vol.1, $\mathrm{n}^{\circ} 2$, pp.211-222.

KaUfman Edy (1976), «La política exterior de la Unidad Popular chilena", Foro Internacional, Vol. 17, No. 2 (66) (Oct. - Dec.), pp. 244-274

MuÑoz, Heraldo (1984), «Las relaciones exteriores del gobierno militar chileno», en Puig, Juan Carlos (Comp.), América
Latina: Políticas Exteriores Comparadas, Buenos Aires: GEL.

Muñoz, Heraldo (2008), A Solitary War. A Diplomat's Chronicle of the Iraq War and Its Lessons, Golden, Colo., Fulcrum, 270 pp.

Rumié, Fuad(2009), «El Estado chileno ante el conflicto en Palestina", Hoja de Ruta, $\mathrm{n}^{\circ} 25$, Julio, http://www.hojaderuta.org/ ver_articulos.php?id_texto=4668id_revista=35 (25/09/2011).

Sepúlveda, Andrés (1991), «Nuestros vínculos con África negra: pasado y presente», Diplomacia, n54-55, pp.9-13.

Shain, Yossi (1999), Marketing the American Creed Abroad, Diasporas in the US and their Homelands, Cambridge University Press, Cambridge, 294 pp.

SHARIF, Regina (1977), "Latin America and the Arab-Israeli Conflict», Journal of Palestine Studies, vol.7, n¹, Autumn pp. 98-122.

VAGNi, Juan José (2005), «Brasil y la Cumbre América del Sur-Países árabes: ¿Encuentro estratégico o diplomacia de fanfarria?», Relatos del Sur, vol.1, N¹, pp.101-128.

VAGNI, Juan José (2009), «La cumbre América del Sur-Países Árabes (ASPA): Balances de un acercamiento estratégico", Revista de Estudios Internacionales Mediterráneos, $\mathrm{n}^{\circ} 8$.

Wilhelmy, Manfred y Roberto, Durán (2003), «Los principales rasgos de la política exterior chilena entre 1973 y el 2000», Revista de Ciencia Política, Vol. XXIII, no 2 , pp. 273-286.

Yopo Herrera, Boris (2010), «La política exterior del gobierno de Piñera: el discurso y las primeras decisiones", Análisis y Propuestas - Política Internacional, Santiago, Friedrich Ebert Stiftung-Chile.

\section{BOLETINES OFICIALES}

BCN Informe, «Estado de Israel: características políticas y relación bilateral con Chile», 25 de febrero de 2011.

BCN Minuta, «Posición de Chile frente al conflicto palestino-israelí y relación bilateral chileno-palestina», 18 de enero de 2011. 
Dirección General de Relaciones Económicas Internacionales (DIRECON), "Comienza misión al Golfo Pérsico", 26 de enero de 2007.

DIRECON, «Alimentos chilenos conquistaron paladares en Dubái», $1^{\text {ro }}$ de marzo de 2007. DIRECON, "Directora de ProChile inaugura oficina comercial en Dubai», 20 de mayo de 2008.

DIRECON, «ProChile desarrolla positiva misión a mercados de Medio Oriente», 5 de junio de 2008.

DIRECON, «Chile y Emiratos Árabes Unidos firman acuerdo de cooperación econocia», 20 de octubre de 2009.

DIRECON, «Nuevas oportunidades de negocios para compañías chilenas en Dubái», 21 de octubre de 2009.

DIRECON, «Inversión directa de capitales chilenos en Medio Oriente 1990 - Diciembre 2009», octubre de 2010.

Ministerio de Relaciones Exteriores de Brasil (MRE), «Explicação de voto do Brasil na Sessão Especial do Conselho de Segurança sobre a Situação na Líbia - Nueva York, 26 de febrero de 2011» $\left(\mathrm{n}^{\circ} 79\right), 28$ de febrero de 2011.

Ministerio de Relaciones Exteriores de Chile (MINREL), Memorias anuales 2005, 2006. MINREL, «Ministro inauguró Embajada de Chile en Emiratos Árabes», 18 de octubre de 2009. MINREL, "Declaración del Gobierno de Chile sobre el reconocimiento del Estado de Palestina", 7 de enero de 2011.

DTO 1866, RREE, 1996.

DS 57, RREE, 1994.

\section{PRENSA}

«El Vice canciller chileno Enrique Valdés Puga en Jordania», El Mercurio (Santiago), 15 de agosto de 1975.

«L'État palestinien reconnu par une cinquantaine de pays", Le Monde (Paris), 25 de noviembre de 1988.

"Cámara de diputados recibió a representantes palestinos", La Segunda (Santiago), 17 décembre 1992.
"'Pionero de una fascinante aventura diplomática', Habla primer embajador de Chile en Palestina", La Tercera (Santiago), 17 de junio de 1998 .

"Mea culpa de Lagos a empresarios árabes», La Segunda (Santiago), 19 de abril de 1999. «Presidente Lagos llama a fortalecer el multilateralismo", El Mercurio (Santiago), 22 de septiembre de 2004.

"Chile como miembro observador permanente de la Liga Árabe», Al Damir (Santiago), $\mathrm{n}^{\circ} 42$, marzo de 2005, p.16-21.

«Rice elogia 'crescente papel global' do Brasil», BBC Brasil (Brasilia), 27 de abril de 2005

«Summit with Arab Countries Turns Political», IPS News, 11 de mayo de 2005, http://www.ipsnews.net/africa/interna. asp? idnews=28646 (25/09/2011)

"Seminario Seeds for the future. Exitosa cumbre árabe-latinoamericana de Fundación Belén 2000", Al Damir (Santiago), n63, octubre de 2007, p.12-19.

«El rey midas en acción», La Nación (Santiago), 4 de noviembre de 2007.

"Viera-Gallo visitará a Shimón Peres y Mahmoud Abbas", La Nación (Santiago), 14 de noviembre de 2008.

«Cámara pide al Gobierno promover cese al fuego en la Franja de Gaza», El Mercurio (Santiago), 7 de enero de 2009.

"Chile busca acordo com o Golfo", ANBA (São Paulo), 25 de marzo de 2009.

«Bachelet asistirá a cumbre de Doha como Presidenta de Chile y de Unasur», Radio Cooperativa (Santiago), 30 de marzo de 2009.

«Mahmud Abbas es recibido por Bachelet con honores", La Nación (Santiago), 25 de noviembre de 2009.

«Senado aprueba en forma unánime proyecto que condena ataque israelí», El Mercurio (Santiago), 1 de junio de 2010.

«Lagos recuerda su oposición a la guerra de Irak tras el fin de la misión militar de Estados Unidos en ese país», El Mercurio (Santiago), 3 septembre 2010.

«The United States and Latin America: Nobody's backyard", The Economist (Londres), 9 de septiembre de 2010. 
Cecilia Baeza y Elodie Brun • La diplomacia chilena hacia los países árabes...

«EEUU rechaza que en Sudamérica se reconozca al Estado palestino", La Nación (Santiago), 8 de diciembre de 2010 .

«Parlamentarios de todos los sectores pidieron al Gobierno reconocer al Estado palestino", Radio Cooperativa (Santiago), 20 de diciembre de 2010.

«El efecto Gadafi en América Latina», Infolatam (Madrid), 28 de febrero de 2011.
«Piñera inicia histórica primera visita de un Presidente chileno a Palestina», El Mercurio (Santiago), 5 de marzo de 2011.

«Chile: Piñera hace un llamamiento en Israel a favor de la paz», Efe (España), 6 de marzo de 2011. 